\title{
Diagnóstico del Sistema Español de Innovación: agentes, recursos y resultados
}

\section{Diagnosis of the Spanish Innovation System: agents, resources and results}

Pablo Galaso*

\begin{abstract}
Resumen
El presente artículo analiza la evolución reciente del sistema español de innovación en comparación con el de los principales países de su entorno. Para ello, describe el papel desempeñado por sus agentes, así como los recursos destinados a la I+D y los resultados obtenidos. Los datos muestran una considerable mejora, especialmente en aspectos relacionados con el desempeño del sector público y la evolución de las publicaciones científicas. No obstante, se observa todavía un importante atraso e ineficiencia en otros ámbitos como la implicación del sector empresarial, el registro de patentes o el comercio exterior de productos tecnológicos.
\end{abstract}

Palabras clave: innovación, desarrollo tecnológico, Sistema Nacional de Innovación, patentes.

\begin{abstract}
This paper analyzes the recent evolution of the Spanish innovation system compared to that of the leading innovative countries. To do so, it describes the role played by its agents, the resources employed in R\&D activities and the outputs obtained by the system. The data analyzed show considerable improvements, especially regarding the development of innovation in the public sector and the increase of scientific publications. However, the study reveals also important delays and inefficiencies in other aspects such as private firms' implication on R\&D activities, patents registration or technological products' exports.
\end{abstract}

Keywords: innovation, technological development, National Innovation System, patents. 


\section{Introducción}

El amplio consenso académico acerca de la relación entre innovación y crecimiento económico otorga una enorme relevancia a los sistemas nacionales de innovación. En el caso español, la actividad innovadora ha mostrado durante los últimos años un esfuerzo y resultados notablemente inferiores a los de las naciones de su entorno.

El conocimiento y diagnóstico de esta desventaja se convierte en requisito indispensable para afrontar adecuadamente el problema, y esto resulta especialmente relevante ante la situación actual de la economía española. La crisis iniciada en 2008 y sus devastadoras consecuencias para el país han puesto de manifiesto la necesidad de cambiar el modelo productivo español -tradicionalmente basado en la construcción y el turismo- de modo que evolucione hacia sectores de mayor valor ańadido (Myro, 2011).

Esto implica, necesariamente, una atención especial a las tareas de innovación, a la forma en que se han llevado a cabo hasta ahora y a las posibilidades de desarrollo en el futuro. Por tanto, el presente trabajo pretende ofrecer un panorama global y realista del Sistema Español de Innovación (SEI) en comparación con el de los principales países de su entorno, naciones de la Unión Europea (UE) y otros referentes en el seno de la ocDE. Además, el artículo señala sus principales logros así como los aspectos en donde resulta más urgente mejorar.

En el caso español, el sistema de innovación se ha caracterizado a lo largo del último siglo por mostrar un esfuerzo y capacidad de generación interna de tecnología notablemente inferiores a los de los países occidentales más avanzados. De hecho, sus debilidades en absoluto se corresponden con la positiva evolución que España ha logrado en otros aspectos de su realidad económica y social (Molero, 2013).

Con la llegada de la democracia, a partir de 1978, España comenzó a experimentar un importante proceso de convergencia hacia los países de su entorno. Proceso que se refleja tanto en los recursos que la economía ha dedicado a la $\mathrm{I}+\mathrm{D}$, como en los resultados que estas actividades han ido obteniendo a lo largo de los años; sin embargo, dado el significativo atraso de partida, esta convergencia ha sido insuficiente, lo que ha impedido cerrar la brecha existente con los principales países desarrollados. En otras palabras, aunque el SEI evoluciona de forma positiva, actualmente sigue mostrando importantes atrasos y debilidades.

Este artículo trata de mostrar dichas carencias y, en su caso, descubrir los puntos fuertes. Para ello, tras una revisión bibliográfica, analiza el papel que desempeñan los principales agentes que intervienen en el Sistema Nacional de Innovación; es decir, las empresas, el Sistema Público 
de Innovación, las administraciones públicas, las organizaciones de soporte a la innovación y el entorno general. Tras esto, se describen los indicadores más relevantes relacionados con los recursos destinados a la innovación y los resultados obtenidos por el sistema en España, y se analizan de forma comparativa con los países de su entorno. Concretamente se estudian datos de gastos y personal destinado a I+D, publicaciones científicas, registros de patentes y comercio exterior de productos tecnológicos. Finalmente, y a modo de conclusión, el último punto ofrece un diagnóstico de la situación, incidiendo en las debilidades más importantes del sistema.

\section{Fundamentos teóricos y metodológicos}

El concepto de sistema de innovación fue introducido en la literatura académica por Lundvall (1985) y, desde entonces, se utiliza ampliamente para sistematizar los elementos e interacciones que intervienen en los procesos de innovación. Su estudio se ha llevado a cabo, principalmente, desde dos perspectivas: una sectorial, que analiza la innovación en distintas ramas de actividad económica (Breschi y Franco, 1997), y otra geográfica, que se centra en las diferencias innovadoras entre regiones (Cooke, 1996) o entre países (Freeman, 1987). Sobre la base de esta última perspectiva -sin duda la más estudiada- diversos autores trabajan con el concepto de Sistema Nacional de Innovación (véanse, por ejemplo, Freeman, 1987; Lundvall, 1992; Nelson, 1993 o Edquist, 1997).

Así, se entiende por Sistema Nacional de Innovación al conjunto de elementos y relaciones, localizadas en el interior de una nación, que interactúan para la producción, difusión y uso de nuevos conocimientos económicamente útiles (Lundvall, 1992). Esta visión parte de tres premisas (Johnson et al., 2003): (1) los sistemas nacionales difieren en su especialización productiva, comercial y de conocimiento; (2) aquellos elementos relacionados con el conocimiento, que son relevantes para el desempeńo económico, están localizados y difícilmente pueden desplazarse de un lugar a otro; (3) la interacción entre agentes resulta indispensable para la adecuada innovación.

A pesar de algunas dificultades en su aplicación, estas premisas facilitan la comprensión de los procesos de innovación y ofrecen explicaciones relevantes a las divergencias en el crecimiento económico y la productividad entre las naciones. Por ello, el estudio de los sistemas nacionales de innovación es ampliamente abordado tanto en investigaciones estrictamente académicas como en publicaciones institucionales que describen y comparan la innovación en diversos países (por ejemplo: Nelson, 1993; OCDE, 1997 o National Science Foundation, 2012). 
En términos metodológicos, estos estudios identifican a los elementos o actores más relevantes del Sistema Nacional de Innovación y emplean una serie de indicadores para medir su desempeño. El presente trabajo aplica esta metodología al caso español y, con este fin, analiza cinco actores principales: las empresas, el sector público, las administraciones públicas, los organismos de soporte a la innovación y el entorno (Fundación Cotec, 2004).

Primero, como unidades básicas de producción, las empresas se encargan de ofrecer bienes y servicios al mercado. Por tanto, el proceso de innovación desempeña en ellas un papel determinante en términos de competitividad y productividad. Esto implica que las innovaciones se llevan a cabo principalmente en su seno y, especialmente, tras la interacción de unas con otras; lo que las convierte en el elemento central de todo Sistema Nacional de Innovación (Lundvall, 2007).

Segundo, el Sistema Público de Innovación aglutina al conjunto organismos de titularidad pública, tales como universidades y centros públicos de investigación, dedicados a las actividades de I+D (Fundación Cotec, 2004). Su papel dentro del sistema nacional es doble: por un lado se encarga de formar a los futuros investigadores y, por otro, de generar directamente conocimiento científico en conexión con las necesidades de la sociedad (OCDE, 1997).

Tercero, el entramado institucional y legislativo de las administraciones públicas es el que regula las actividades innovadoras, ordena el Sistema Público de Innovación, coordina las políticas públicas y fomenta la actividad innovadora de las empresas desde distintos ámbitos. Por ello, se considera esencial en todo Sistema Nacional de Innovación por tres razones: porque reduce la incertidumbre ofreciendo información, gestiona conflictos y acuerdos entre agentes, y ofrece incentivos adecuados para la innovación (Edquist y Johnson, 1997).

Cuarto, las organizaciones de soporte a la innovación incluyen a todas aquellas entidades encargadas de apoyar a las empresas innovadoras. Sus tareas consisten en la provisión de medios materiales y humanos dedicados directamente a la $\mathrm{I}+\mathrm{D}$, así, como la ayuda en cuestiones técnicas, de gestión, de información o de otro tipo relacionadas con el proceso de innovación de las empresas (Edquist y Johnson, 1997).

Por último, el entorno está formado por una serie de componentes que, aunque no dedican su actividad específicamente a la innovación, sí pueden influir en la I+D desarrollada por los otros agentes. Los elementos del entorno que más condicionan los procesos de innovación son: los mercados de bienes y servicios, los mecanismos de financiación y el capital humano del país (Fundación Cotec, 2004). 
En lo que respecta a los indicadores, la literatura académica suele distinguir entre los recursos destinados al Sistema Nacional de Innovación y los resultados obtenidos por el mismo -es decir, sus inputs y outputs(OCDE, 1997). Así, para medir los recursos se emplea, principalmente, el gasto y el personal destinado a I+D; mientras que para cuantificar los resultados se observa el número de artículos científicos, las patentes o el comercio de productos con alto contenido tecnológico. Todos estos indicadores presentan ventajas e inconvenientes; sin embargo, su combinación permite ofrecer un diagnóstico amplio y adecuado del Sistema Nacional de Innovación (Katz, 2006).

\section{Agentes del sistema español de innovación}

A continuación estudiaremos el papel desempeñado por las empresas privadas, la Administración Pública, el sistema público de I+D y las organizaciones de soporte a la innovación en España. Además, describiremos el entorno en el que actúan estos cuatro agentes.

\subsection{Las empresas}

En el caso de la economía española, las empresas representan el eslabón más débil del sistema. Así, en el índice sintético de innovación elaborado por Eurostat (2014), los indicadores relacionados con el desempeño empresarial ofrecen sin lugar a dudas los peores resultados en términos comparativos. ${ }^{1}$

Con más o menos énfasis, diversos diagnósticos de investigaciones anteriores coinciden en la debilidad que supone para el SEI, el sector empresarial (Buesa, 2003; Beraza y Rodríguez, 2008; Cañibano y Castro, 2010; Molero, 2010). Las principales razones de esta debilidad son la escasa cultura y tradición innovadora de las empresas, el bajo perfil educativo de sus trabajadores, la reducida cooperación empresarial en materia de innovación y los exiguos niveles de creación de empresas de base tecnológica.

Respecto a la primera razón, la menor cultura innovadora implica que las empresas españolas estén poco propensas a emplear la tecnología como instrumento de competitividad y de mejora de la productividad. Concretamente, y según datos del INE (2012a), la proporción que representan las empresas innovadoras (es decir, el porcentaje de aquellas que, en su ciclo productivo, llevan a cabo innovaciones de cualquier tipo) respecto

\footnotetext{
${ }^{1}$ El Índice Sintético de Innovación (Summary Innovation Index) se calcula empleando múltiples indicadores relacionados con la innovación y agrupados en tres dimensiones: facilitadores, actividades empresariales y resultados (Eurostat, 2014).
} 
del total de empresas, se situaba en 2012 en torno al 13 por ciento. Además, este porcentaje viene reduciéndose progresivamente desde 2004, cuando alcanzaba cerca del 30 por ciento.

Al comparar la propensión innovadora de las empresas con la media de la uE observamos que, según los resultados de la Encuesta Europea de Innovación (Eurostat, 2005), la proporción de empresas innovadoras se sitúa en España doce puntos porcentuales por debajo de la media europea. Además, su intensidad innovadora (medida como el porcentaje de gastos de innovación respecto de la cifra de negocios) es también muy inferior a la de las empresas de las economías de su entorno, situándose en $60 \%$ de la media europea. Junto a esta característica, se puede constatar que existe una gran concentración de recursos dedicados a la $\mathrm{I}+\mathrm{D}$ en unos pocos sectores, especialmente en la industria química, la informática y los servicios de $\mathrm{I}+\mathrm{D}$, lo que pone de manifiesto que, para la gran mayoría de las actividades económicas, los recursos empresariales dedicados a la innovación son prácticamente marginales (Molero, 2007).

En cuanto a las pautas de innovación, ${ }^{2}$ si nos atenemos a la tipología elaborada por Eurostat (2005), se podrían distinguir cuatro tipos de empresas:

a) Innovadores estratégicos: empresas para las que la innovación es un componente de la estrategia competitiva, por lo que desarrollan actividades de I+D de forma continua. Suelen ser la principal fuente de innovación que, más tarde, se difundirá por el tejido empresarial de una economía.

b) Innovadores intermitentes: empresas para las que la innovación no forma parte de su actividad estratégica, por lo que la llevan a cabo únicamente cuando es necesario o favorable para su actividad empresarial. Habitualmente sus actividades de $\mathrm{I}+\mathrm{D}$ van dirigidas a adaptar, a su modelo de negocio, las innovaciones desarrolladas por otras empresas.

c) Modificadores de tecnología: empresas que, mediante la I+D, modifican productos o, especialmente, procesos productivos propios.

d) Adoptantes de tecnología: empresas que llevan a cabo una estrategia de adopción de las innovaciones desarrolladas por otras empresas u organizaciones.

$\mathrm{Al}$ analizar el porcentaje de empresas de cada tipo y comparar los resultados espańoles con la media de la UE (figura I), se descubre que en España la mayor parte de empresas innovadoras son adoptantes de tecnología, mientras que en la UE este tipo de empresas representa una

${ }^{2}$ Las pautas de innovación de las empresas se deducen de la composición de sus gastos de innovación. Las dos pautas principales, que se traducen en las partidas más importantes del gasto total, pueden resumirse como: (1) aquellas que van dirigidas a crear tecnología propia, mediante la realización de actividades de I+D interna o externa, y (2) las que adquieren tecnología a terceros, mediante la compra de equipos con tecnología incorporada o la adquisición de conocimientos externos (Eurostat, 2014). 
proporción mucho menor. En el otro extremo, la proporción de empresas identificadas como innovadores estratégicos es en España mucho menor que en la Ue. Todo ello pone de manifiesto el atraso español no sólo en cantidad, sino también en la calidad de la innovación (Molero, 2007), al constatarse que la mayor parte de las empresas únicamente adoptan innovaciones desarrolladas por otros y sólo un porcentaje marginal de ellas basa su estrategia competitiva en la innovación.

\section{Figura I}

Tipos de empresas innovadoras en España y en la UE (En \% sobre el total de empresas)

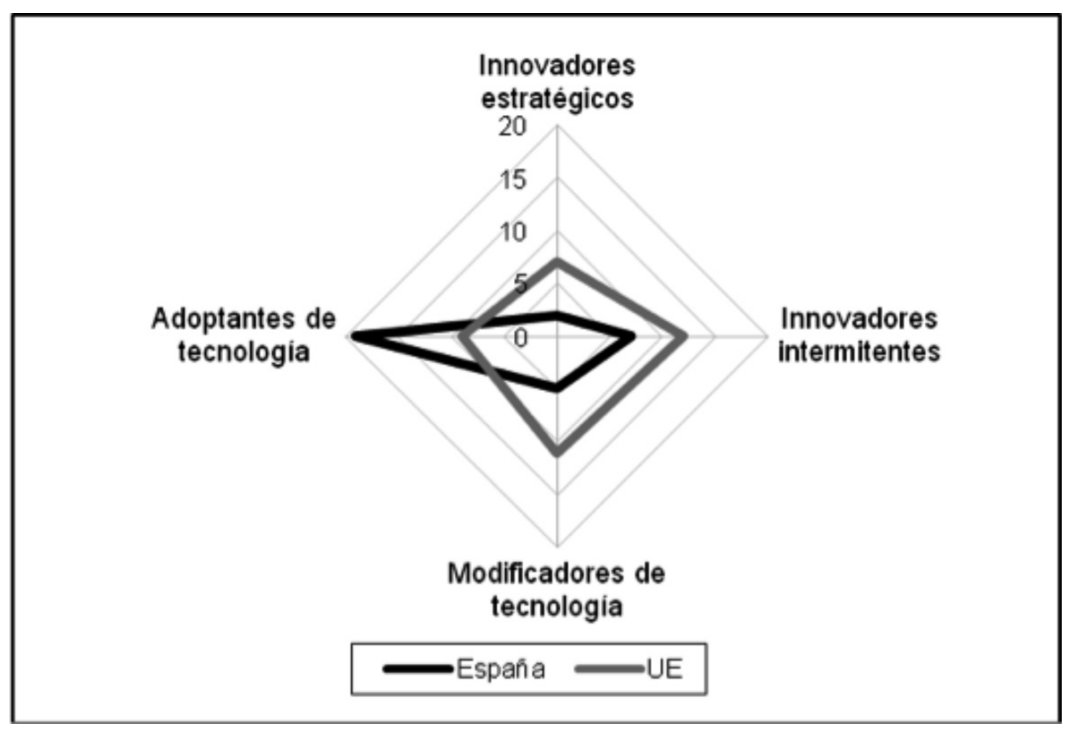

Fuente: Eurostat (2005).

El segundo diagnóstico acerca del desempeño innovador de las empresas se refiere a su capital humano. En este aspecto, tanto el bajo perfil educativo de los trabajadores como los reducidos esfuerzos empresariales para la formación continua de sus empleados mantienen un capital humano poco propicio para impulsar la innovación en las empresas (PlanasColl, 2005).

Según Eurostat, en 2005 sólo 47\% de las empresas españolas desarrollaban actividades de formación continua de su personal, frente a $62 \%$ de las empresas europeas. Además, estas actividades de formación supusieron en España un coste de 1.2\% del gasto salarial, mientras que en la UE este dato se situó en 1.6\% (Eurostat, 2005). A este hecho contribuye 
también la alta tasa de temporalidad en el empleo del mercado laboral español.

El tercer diagnóstico se refiere a las relaciones que establecen las empresas para llevar a cabo actividades de innovación. Con el fin de afrontar eventuales limitaciones en recursos humanos o financieros, las empresas pueden colaborar con centros públicos u otras empresas y beneficiarse de las ventajas que suponen los proyectos de innovación conjunta. En este aspecto, los trabajos realizados por Cañibano y Castro (2010) y Navarro (2002) inciden especialmente en la escasa colaboración entre empresas innovadoras. Las encuestas realizadas muestran que el tejido empresarial español es más bien reacio a la interacción en las labores de innovación y se decanta más por proyectos aislados (Fundación SEPI, 2009). Esto impide al Sistema Nacional de Innovación beneficiarse adecuadamente de la interacción entre agentes.

Finalmente, la cuarta razón del atraso empresarial se refiere al bajo nivel de creación de empresas tecnológicas. Lo que pone de relieve la lentitud en la modernización del tejido productivo y empresarial español. Como muestran Beraza y Rodríguez (2008), el peso de las actividades de alta tecnología dentro del sector manufacturero está muy por debajo de la media europea tanto en el número de empresas, como en el valor y el empleo añadido que generan.

\subsection{El Sistema Público de Innovación}

La configuración actual del Sistema Público de Innovación en España es fruto de los cambios vividos a partir de los años ochenta. Así, su evolución positiva en términos de formación de investigadores y de producción científica de calidad ha permitido que en la actualidad sea comparable a los sistemas públicos de innovación de los países de su entorno. Por ello, coincidimos con el diagnóstico de Molero (2010) identificando al sector público como el actor con el mejor desempeño del SEI.

Concretamente, según Eurostat (2005), los investigadores de este sector representaban en España $0.64 \%$ de la población activa en 2003, por encima de la media de la Ue-25 (con 0.5\%) y de países como Alemania o Francia (con 0.55 y 0.48 por ciento, respectivamente). Asimismo, aunque el gasto en $\mathrm{I}+\mathrm{D}$ ejecutado por el sector público tradicionalmente ha estado por debajo de la media europea (gráfica I), la tendencia reciente muestra un acercamiento a los estándares de los países desarrollados.

Sin embargo, sus resultados no han sido tan positivos en lo que respecta a la generación de conocimientos aplicables a las empresas. Este hecho se debe a varias debilidades que presenta el Sistema Público de Innovación en España, entre las que destacamos las tres siguientes (Fun- 


\section{Gráfica I \\ Gastos en I+D ejecutados por el sector público}

(En \% del PIB)

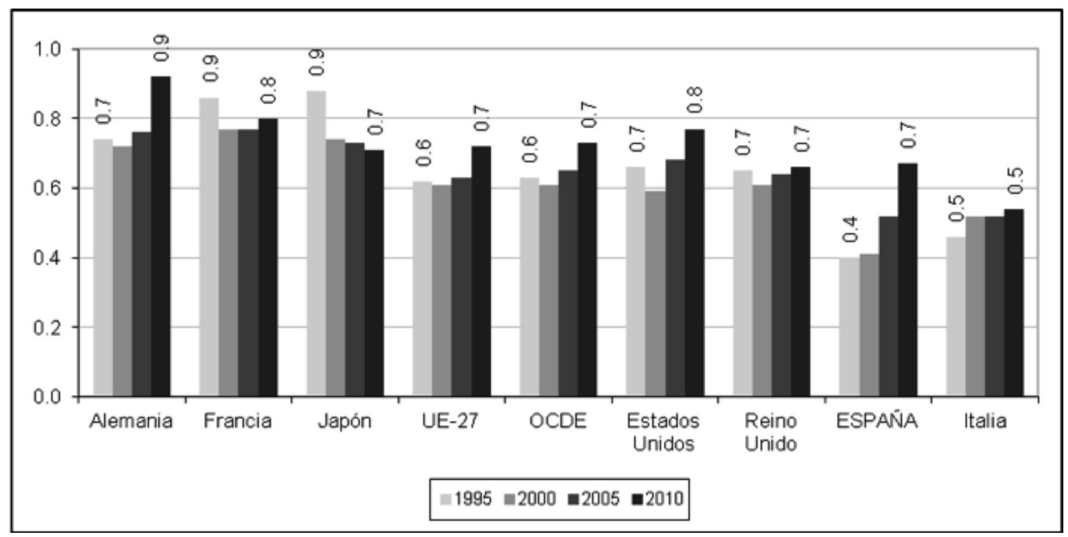

Fuente: OCDE (2008 y 2013).

dación Cotec, 2011), en primer lugar, los grupos de investigación aplican con dificultad sus resultados en el tejido empresarial debido a su reducida dimensión, sus bajos niveles de colaboración interdisciplinar y su escasez de personal de apoyo.

En segundo lugar, la exigua implicación del sector público en la creación de empresas de base tecnológica tampoco ha fomentado el vínculo con el sistema productivo. Finalmente, las limitaciones que presentan las organizaciones encargadas de facilitar la transferencia de resultados de la investigación (tales como las Oficinas de Transferencia de Tecnología), dado su reducido tamaño y su especialización, obstaculizan enormemente su labor de cooperación y transferencia de los resultados del sistema público al tejido empresarial. Este último aspecto lo trataremos posteriormente a detalle.

\subsection{Las administraciones públicas}

Desde 1986 los recursos destinados por la Administración General del Estado al fomento de la actividad innovadora han mostrado una tendencia creciente, lo que indudablemente ha contribuido al acercamiento del SEI al de los principales países desarrollados (véase la evolución del Gasto público en $\mathrm{I}+\mathrm{D}$ presentada en la gráfica I). El instrumento más importante para la canalización de esos fondos, tanto los nacionales como los provenientes de los Fondos Estructurales de la UE, ha sido el Plan Nacional de I+D. 
Sin embargo, en esta evolución ascendente se observan algunos aspectos disonantes. Por un lado, desde finales de los años noventa, se puede constatar que el crecimiento de los recursos se ha debido principalmente a los créditos a la innovación, más concretamente, a los dedicados a la investigación militar, lo que separa al caso español de la distribución -más adecuada- de los recursos públicos registrada por los principales países de la OCDE. ${ }^{3}$

Por otro lado, aunque las ayudas fiscales a las empresas sean generosas, existe un amplio margen de mejora en este ámbito, especialmente en lo que respecta a las definiciones fiscales de actividades de innovación, el tratamiento de las pymes y el de los gastos realizados en el exterior (Rivas, 2007). Así, las modalidades de los fondos otorgados para la investigación, la excesiva burocracia en los criterios de selección, los controles reiterados y los avales exigidos para acceder a las subvenciones no han logrado atraer a las empresas en la medida necesaria para que los programas de fomento contaran con una mayor implicación y gasto privado en las actividades de I+D (Fundación Cotec, 2004).

En cuanto a la regulación de las actividades de innovación, el marco jurídico español ha contribuido en las últimas décadas a fortalecer al Sistema Público de Investigación, a aumentar la infraestructura y el número de investigadores así como a incrementar la producción de publicaciones científicas (López-Facal et al., 2006). No obstante, dicho marco jurídico sigue mostrando algunos aspectos mejorables.

En primer lugar, destaca su complejidad en la distribución de competencias, fruto del marco constitucional en que se encuentra inscrito. Como señala Tortosa (2006), aún no se ha conseguido establecer un mecanismo de cooperación y coordinación que contemple todas las políticas de ciencia y tecnología del Estado, y de éstas con la Unión Europea.

En segundo lugar, la Ley de la Ciencia de 1986 -marco regulatorio fundamental de las actividades de I+D- presenta algunas deficiencias importantes (López-Facal et al., 2006): falta de orientación a medio y largo plazo, exceso en el número de proyectos (lo que fomenta la atomización, la dispersión de esfuerzos y la disminución de capacidades) y, finalmente, falta de articulación entre sector público y privado y entre regiones espańolas. Todo esto supone una importante pérdida de oportunidades para el SEI en su conjunto.

\footnotetext{
${ }^{3}$ En España, las ayudas del Estado en forma de créditos para la innovación han pasado de representar únicamente $5 \%$ del total de los fondos en 1997 a más de $50 \%$ en 2004. Ese mismo año, en los países de la ocde esta cifra fue únicamente de 20\% del total (Fundación Cotec, 2004).
} 


\subsection{Las organizaciones de soporte a la innovación}

Los principales organismos de este tipo que operan en España son los centros tecnológicos, las organizaciones de transferencia de tecnología y de sensibilización hacia la tecnología, ${ }^{4}$ los centros de modernización tecnológica para el aumento de productividad y los parques científicos y tecnológicos.

Aunque en España existen numerosas organizaciones de este tipo enormemente eficientes en el desempeño de sus funciones, muchas otras no han logrado aún integrarse adecuadamente en el SEI. Asimismo, la distribución territorial de estos centros es considerablemente desigual, por lo que aparecen grandes diferencias entre las comunidades autónomas (Cañibano y Castro, 2010).

Respecto a su actuación, la mayor parte de los centros tecnológicos se dedican a ofrecer servicios de soporte en tecnología avanzada, descuidando la asistencia a las empresas en la modernización de sus procesos productivos y de gestión de la tecnología. Por otro lado, las organizaciones de transferencia y de sensibilización hacia la tecnología se ciñen casi exclusivamente al desarrollo de tareas administrativas y de identificación de recursos, así como al conocimiento de sus instituciones, sin asumir adecuadamente responsabilidades relacionadas con la difusión y comercialización de las innovaciones. Estos factores dificultan considerablemente la aplicación de las innovaciones generadas por el sistema de innovación al tejido productivo y empresarial (Fundación Cotec, 2011).

En cuanto a los centros de investigación españoles, su papel se ha reforzado notablemente en las últimas décadas en términos de sus recursos humanos y financieros, así como en su dotación de infraestructuras y equipos, lo que sin duda ha contribuido a mejorar sustancialmente la producción científica española (Cañibano y Castro, 2010); sin embargo, salvo en algunas excepciones, su dimensión y relevancia son todavía muy reducidas en comparación con sus homólogos de los principales países europeos.

Finalmente, respecto a los parques tecnológicos, en los últimos años España está experimentando un considerable surgimiento, desarrollo y modernización de estos espacios (APTE, 2010). Muestra de ello son los datos presentados en el cuadro 1 acerca de la creciente actividad económica registrada en los parques miembros de la Asociación de Parques Científicos y Tecnológicos de España (APTE).

\footnotetext{
${ }^{4}$ Entre ellas, las más conocidas son las Oficinas de Transferencia de Tecnología (OTRI), los Centros de Enlace para la Innovación (CEI), los Centros Europeos de Empresas e Innovación (CEEI) y los recientes centros de difusión tecnológica.
} 


\section{Cuadro 1 \\ Actividad de los parques científicos y tecnológicos miembros de la APTE}

\begin{tabular}{lrrc}
\hline & 2000 & 2010 & $\begin{array}{c}\text { Variación anual } \\
\text { media 2000-2010 } \\
\text { (\%) }\end{array}$ \\
\hline Número de parques & 23 & 80 & 13.3 \\
Número de empresas & 965 & 5.539 & 19.1 \\
Número de trabajadores & 25.464 & 145.155 & 19.0 \\
Ventas (en millones de $€$ ) & 3.034 & 21.475 & 21.6 \\
\hline
\end{tabular}

Fuente: elaboración propia a partir de datos de APTE (2010).

\subsection{El entorno}

Los tres elementos del entorno que más condicionan los procesos de innovación son los mercados de bienes y servicios, los mecanismos de financiación y el capital humano. En cuanto al primero, se considera que la demanda puede ejercer como mecanismo dinamizador que impulse de algún modo los procesos de innovación de los agentes. A este respecto, algunos trabajos señalan que la especialización productiva característica de la economía española -en sectores de reducida capacidad tecnológicacrea un entorno poco propicio para las actividades de I+D (Buesa, 2003; Beraza y Rodríguez, 2008; Cañibano y Castro, 2010).

En línea con estos argumentos, se observa que, durante los últimos años, España ha aumentado considerablemente la demanda interna de productos de alta y media alta tecnología hasta alcanzar los patrones de la UE. No obstante, esta evolución de la demanda no ha estado acompañada de un impulso adecuado por el lado de la oferta. Por ello, las importaciones de bienes tecnológicos han incrementado también de forma significativa, pasando de 173,2 millones de euros en 2001 a 285 millones en 2007 (INE, 2012b).

En segundo lugar, respecto a los mecanismos de financiación, se observa una consolidación de instrumentos y mercados financieros propios de las actividades de I+D. Así, en la actualidad los fondos de capital riesgo -herramienta muy adecuada para la financiación de la actividad innovadora- han mostrado en España incrementos considerables hasta llegar a una situación comparable a la de la media de la UE; sin embargo, en comparación con los países de su entorno, el capital riesgo español desvía una proporción mayor de sus fondos hacia sectores con menor capacidad tecnológica (ASCRI, 2011) y, por ello, su contribución a la actividad innovadora ha sido menor que en otros países (Cañibano y Castro, 2010). 
El tercer factor con influencia directa en los procesos de innovación es el capital humano. A este respecto, resulta reseńable que la mejora del nivel educativo en las últimas décadas viene de la mano de una fuerte polarización (Cañibano, 2004): en España conviven una proporción muy elevada de personas que no completan los estudios secundarios, un reducido porcentaje de aquellos que cursan ciclos de formación profesional y una gran presencia de titulados universitarios.

Además, según datos de los informes PISA (véase, por ejemplo, OCDE, 2012), España muestra ciertas carencias en comparación con los países de su entorno. En la enseñanza obligatoria, aunque el gasto por estudiante se sitúa en la media de los principales países desarrollados, los resultados no se corresponden con estos niveles; así, los estudiantes en España presentan una capacidad en comprensión lectora y en conocimientos matemáticos y científicos por debajo de la media de los países de la OCDE.

Además, los niveles de abandono escolar tras finalizar la etapa de educación obligatoria son de los más elevados de la UE. Respecto a los estudios de formación profesional, se observa que su importancia relativa dentro del conjunto del sistema educativo español es menor en comparación con la de los países de su entorno. En cuanto a la enseñanza universitaria, aunque el número de estudiantes por millón de habitantes es similar al de la UE, el gasto por estudiante español muy inferior al europeo. Finalmente, se constata una importante carencia en lo que respecta a la formación continua de los trabajadores, ya que la participación de las empresas en programas de este tipo es sustancialmente menor a la de los países más avanzados.

\section{Indicadores del sistema español de innovación}

Una vez estudiados los agentes, nos detendremos en los indicadores más significativos del sistema de innovación. Éstos se pueden dividir en dos grupos diferenciados: recursos y resultados. Los recursos que una economía dedica a las actividades de innovación engloban a los gastos en $\mathrm{I}+\mathrm{D}$ y los recursos humanos destinados a la investigación; en cuanto a los resultados, se observan registros de patentes, indicadores bibliométricos y datos referentes al comercio de productos tecnológicos.

\subsection{Recursos dedicados a la innovación}

Comenzamos analizando los gastos en I+D de la economía española. Desde los ańos noventa, el crecimiento en términos absolutos del gasto español en I+D ha sido muy superior al de los principales países de la oCDE. Así, exceptuando los años entre 1993 y 1997, el gasto total mone- 
tario ha crecido a una tasa anual acumulativa siempre superior a $10 \%$ en Espańa, mientras que en los países de la ocde este ritmo ha estado en torno a 5\% (Fundación Cotec, 2009).

No obstante, esta evolución ha sido insuficiente como para lograr la convergencia absoluta con los líderes mundiales en innovación. Como se observa en la gráfica II, aunque se ha experimentado un cierto acercamiento, Espańa mantiene un nivel inferior de esfuerzo tecnológico medido como proporción del gasto en I+D sobre el PIB. Así, los datos de la economía española se sitúan todavía lejos de la media de la uE y, más aún, de la media de economías de la ocDE.

\section{Gráfica II \\ Gastos totales en I+D \\ (En \% del PIB)}

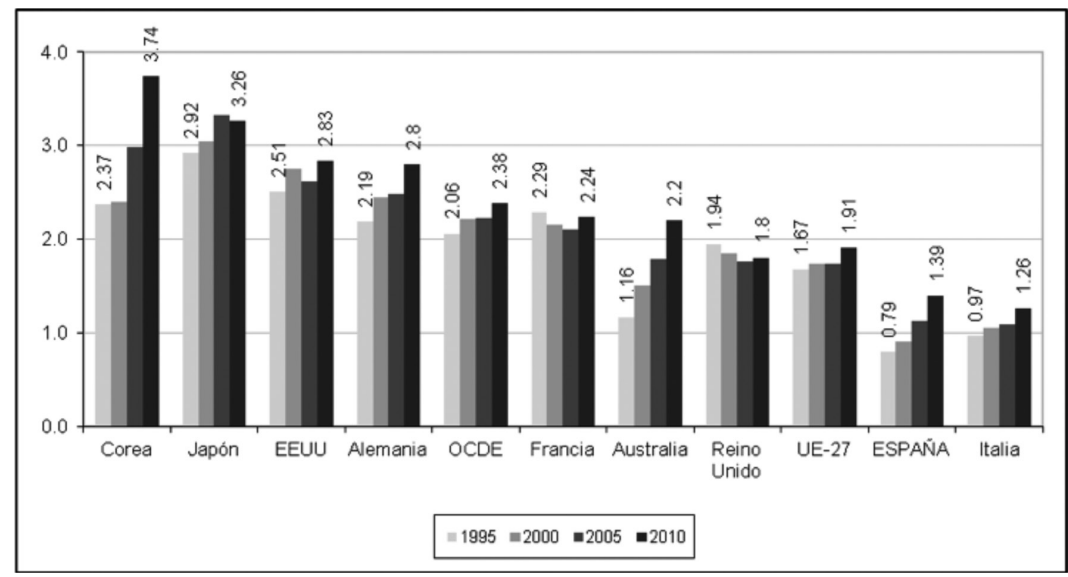

Fuente: OCDE (2008 y 2013).

Respecto a la distribución del gasto en I+D por sector de ejecución, según la OCDE, Espańa ha registrado un importante aumento del peso en el gasto total de las empresas y las instituciones privadas sin fines de lucro (IPSFL), pasando de 44\% en 1981 a 52\% en 2010. Junto a esto, se constata una fuerte reducción del papel de la Administración Pública que, partiendo de $33 \%$ en 1981, se sitúa en $28 \%$ en 2010 . La enseñanza superior se mantuvo en unos niveles alrededor de $20 \%$ hasta el comienzo de la década de los 90; a partir de entonces, su peso aumentó hasta situarse en niveles superiores a 30\% para volver a reducirse hasta $20 \%$ del gasto total en I+D en 2010 .

La evolución experimentada por la distribución del gasto total según los sectores de ejecución ha supuesto un acercamiento a los estándares europeos en la medida en que el peso del gasto empresarial ha crecido 
significativamente. A pesar de ello, el sector empresarial español sigue representando un papel menos relevante que el que tiene en los de países de su entorno. Así, como se observa en la gráfica III, las empresas e instituciones privadas sin fines de lucro (IPSFL) en España fueron responsables de una proporción significativamente menor del gasto total en $\mathrm{I}+\mathrm{D}$ que las de países como Alemania, Francia o Reino Unido. La escasa implicación del sector empresarial supone una de las principales debilidades del SEI.

\section{Gráfica III \\ Distribución de los gastos en $\mathrm{I}+\mathrm{D}$ por sector de ejecución en 2010 (En \% del total)}

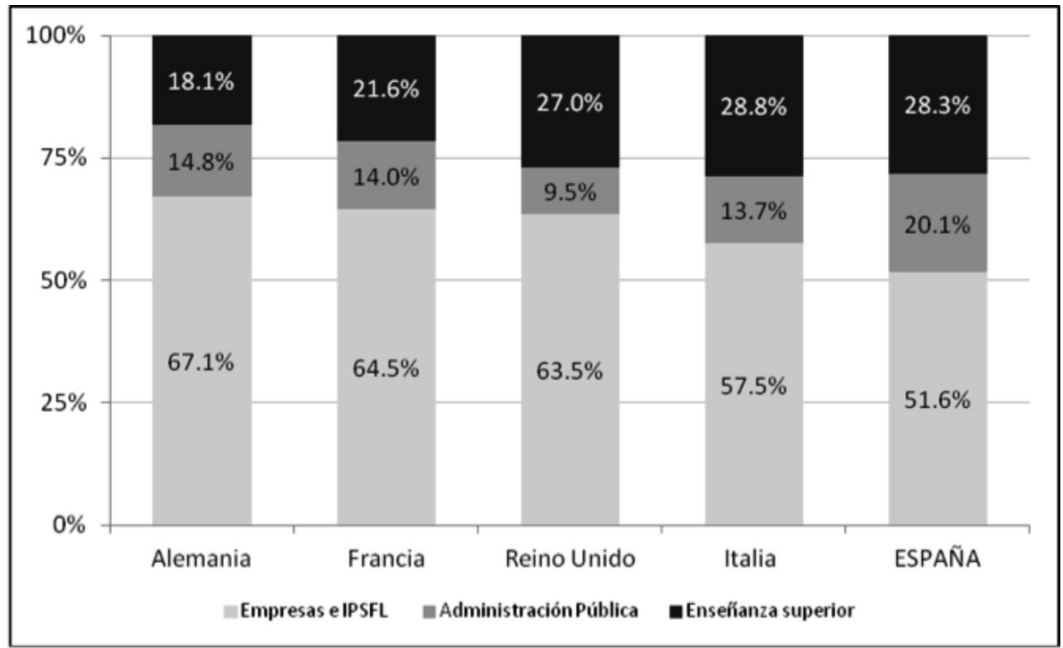

Fuente: OCDE (2013).

Otra característica del Sistema Español de Innovación que se aprecia al analizar los datos de gastos en $\mathrm{I}+\mathrm{D}$ es la diferencia existente entre regiones y la gran concentración de la actividad innovadora en un reducido número de comunidades autónomas. Así, Madrid, Cataluña, Andalucía, País Vasco y Valencia concentran más de $75 \%$ del gasto en I+D español. Asimismo, y aunque su peso se ha reducido proporcionalmente en los últimos años, el papel de Madrid y Cataluña en las actividades de I+D es significativamente superior al resto, llegando a concentrar casi $50 \%$ del gasto total.

$\mathrm{Al}$ analizar la evolución de los recursos humanos dedicados a la innovación, se observa un crecimiento considerable desde los ańos 80 , tanto en el número de investigadores como en el total de empleados en actividades de I+D. La evolución de este indicador desde entonces ha sido, a 
diferencia del gasto en I+D como porcentaje del PIB, siempre positiva, lo que ha llevado a pasar de los 36.221 empleados en equivalencia a jornada completa de 1981 a más de 200 mil en 2008 (INE, 2012b).

En comparación con los principales países de la UE, el crecimiento del empleo en I+D respecto al total de la población en España ha sido muy superior (gráfica Iv), lo que ha permitido recortar notablemente la distancia o incluso superar a naciones como el Reino Unido o Italia.

\section{Gráfica IV \\ Personal dedicado a actividades de $\mathrm{I}+\mathrm{D}$ por cada mil ocupados}

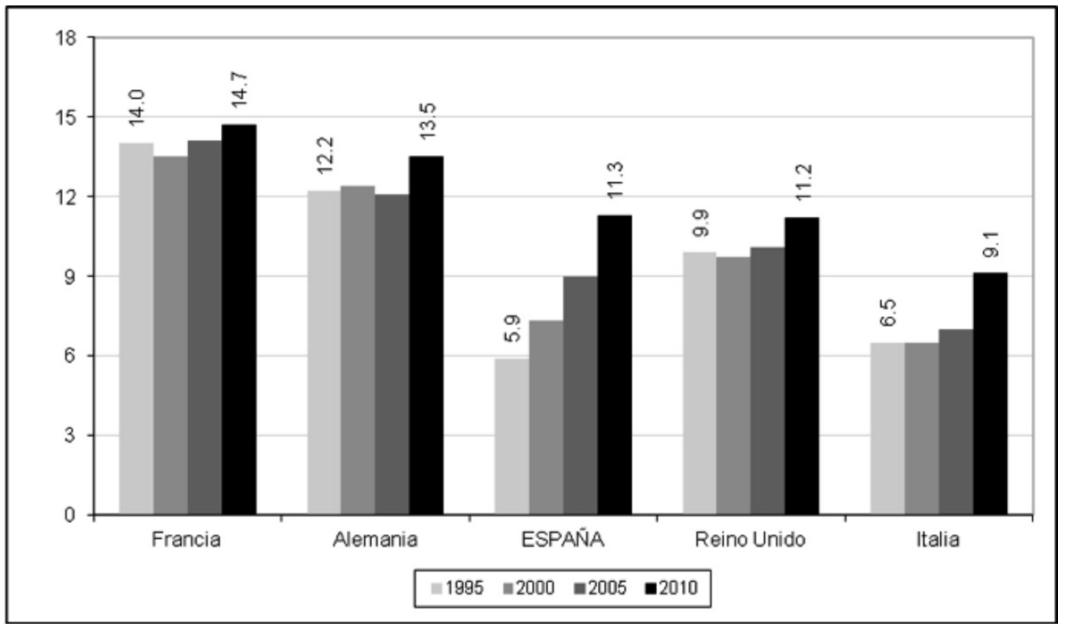

Fuente: OCDE (2013).

Si se desglosa el comportamiento de los recursos humanos empleados en I+D según los distintos sectores de ejecución, se puede observar una mayor presencia de la enseñanza superior (47\% en 2008). No obstante, la tendencia muestra que las empresas e IPSFL han ido acaparando con el tiempo una mayor proporción de investigadores, desde $17 \%$ en 1981 hasta cerca de 35\% en 2009 (INE, 2012b).

Pero el aumento experimentado por el número de investigadores en el sector privado español no ha sido suficiente para equiparar su situación a los cánones europeos (gráfica v). Así, la distribución de la mano de obra investigadora por sector de actividad en España se aleja considerablemente del patrón seguido por países europeos de referencia como Alemania o Francia, que concentran a la mayor parte de estos trabajadores en el sector privado. 


\section{Gráfica $v$}

Distribución del personal dedicado a actividades de $\mathrm{I}+\mathrm{D}$ por sector de ejecución en 2010

(En \% del total)

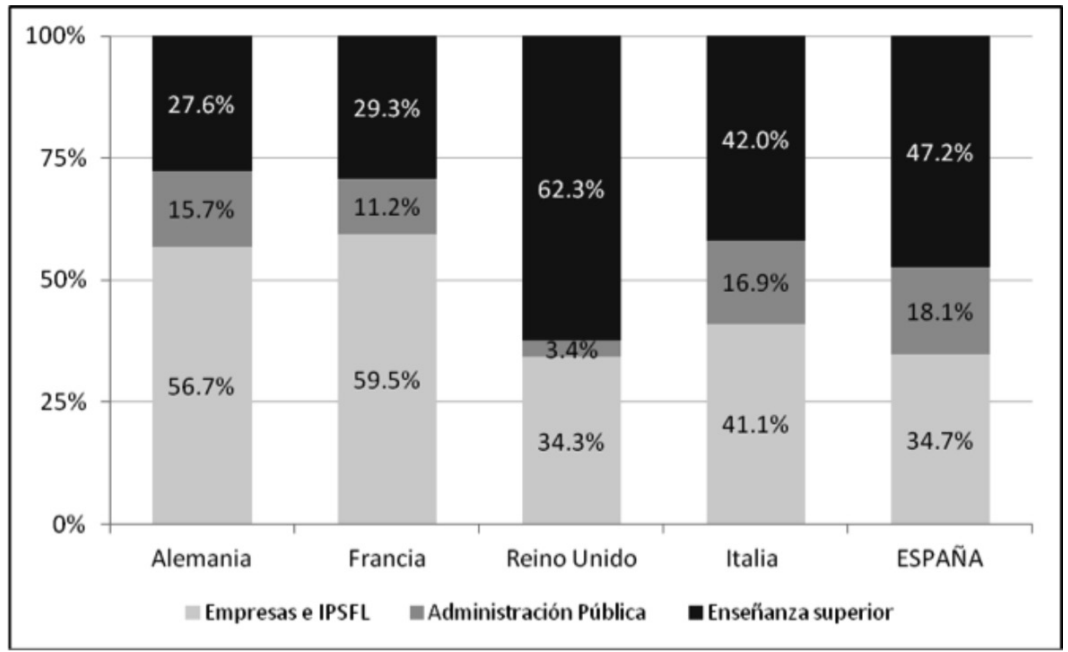

Fuente: OCDE (2013).

\subsection{Resultados de la actividad innovadora}

Comenzamos nuestro análisis de los resultados generados por el Sistema Español de Innovación presentando indicadores referentes a las publicaciones científicas. Estos datos reflejan parte de los resultados obtenidos por un sistema de innovación en la medida en que desempeñan un papel fundamental para la difusión de los conocimientos científicos nuevos.

Atendiendo a los artículos científicos publicados en revistas de difusión internacional englobadas en los índices Science Citation Index (SCI) y Social Sciences Citation Index (ssCI), se puede observar que la producción científica española ha crecido considerablemente en los últimos años (cuadro 2). Así, entre 1999 y 2009, el aumento anual ha sido de 4\% en España, por encima de muchos de los países de su entorno.

Los datos relativos a la productividad científica sitúan a España (con 1446 artículos por millón de habitantes en 2010) ligeramente por detrás de países como Alemania, Francia o Estados Unidos (Fundación Cotec, 2011). No obstante, la evolución experimentada durante los últimos años es mucho más positiva en el caso español. Por ello, los datos relacionados con las publicaciones científicas presentan una imagen del SEI considerablemente mejor que la que ofrecen otros indicadores. 


\section{Cuadro 2 \\ Artículos científicos en revistas de difusión internacional (1999-2009)}

\begin{tabular}{lrrcc}
\hline \multicolumn{1}{c}{ País } & 1999 & 2009 & $\begin{array}{c}\text { Variación anual } \\
\text { media } 1999-2009 \\
(\%)\end{array}$ & $\begin{array}{c}\text { Cuota de producción } \\
\text { mundial, 2009 } \\
(\%)\end{array}$ \\
\hline Estados Unidos & 188.004 & 208.601 & 1.0 & 26.5 \\
China & 15.715 & 74.019 & 16.8 & 9.4 \\
Japón & 55.274 & 49.627 & -1.1 & 6.3 \\
Reino Unido & 46.788 & 45.649 & -0.2 & 5.8 \\
Alemania & 42.963 & 45.003 & 0.5 & 5.7 \\
Francia & 31.345 & 31.748 & 0.1 & 4.0 \\
Canadá & 22.125 & 29.017 & 2.7 & 3.7 \\
Italia & 20.327 & 26.755 & 2.8 & 3.4 \\
Corea & 8.478 & 22.271 & 10.1 & 2.8 \\
ESPANA & 14.514 & 21.543 & 4.0 & 2.7 \\
\hline
\end{tabular}

Fuente: National Science Foundation (2012).

La distribución de la producción científica por regiones españolas muestra unos patrones muy similares a los del gasto en $\mathrm{I}+\mathrm{D}$, es decir, una fuerte concentración en un reducido número de regiones. Así, Madrid, Cataluña, Andalucía y Valencia realizaron 70\% de la producción científica española en 2008 (Moya y Gros, 2010). Si se analiza el número de documentos en función del tamaño de la población, además de las citadas comunidades, destacan otras regiones de pequeño tamaño como Navarra, Cantabria o Aragón, con una elevada producción por habitante.

Otro indicador adecuado para medir los resultados de un sistema de innovación es el de las patentes registradas. En el caso español, conviene agregar los datos de patentes presentadas directamente por vía nacional en la Oficina Española de Patentes y Marcas (oepm) y las que, designando a Espańa, son presentadas en la oficina europea (oep) y en la mundial (OMPI). Al hacer esto, se observa una tendencia de crecimiento continuado y muy significativo desde finales de los años noventa hasta la actualidad, con una tasa anual de crecimiento medio acumulativo cercano al 9 por ciento entre 1997 y 2008, lo que ha permitido casi triplicar el número de solicitudes de patentes (овpM, 2010).

Para situar los resultados españoles de este indicador en el ámbito internacional, conviene tener en cuenta las patentes triádicas, es decir, las solicitadas simultáneamente en la oficina de patentes europea (OEP), la estadounidense (USPTO) y la japonesa (JPO). El análisis de este tipo de 
patentes resulta especialmente ilustrativo, dado que están consideradas como las de mayor valor económico y mayor impacto tecnológico (Sternitzke, 2009).

Como se observa en el cuadro 3, Estados Unidos y Japón concentran más de la mitad de las patentes triádicas concedidas en el mundo. La posición de España es aún muy poco relevante, situándose por debajo del uno por ciento mundial. Estos resultados están en línea con los niveles españoles de gasto en I+D. Por otro lado, si analizamos la productividad a través de los registros de patentes triádicas por millón de habitantes, se observa también que la posición de España sigue siendo muy retrasada no solo con respecto a los líderes mundiales, como Japón o Alemania, sino también con respecto a la media de la Unión Europea.

\section{Cuadro 3 \\ Patentes triádicas concedidas según países (2009)}

\begin{tabular}{lcc}
\hline \multicolumn{1}{c}{ Pais } & En porcentaje del total mundial & Por millón de habitantes \\
\hline EEUU & 29.7 & 45.0 \\
Japón & 27.9 & 101.9 \\
Alemania & 12.0 & 68.2 \\
Francia & 5.1 & 36.9 \\
Corea & 4.3 & 40.9 \\
Reino Unido & 3.4 & 25.9 \\
Italia & 1.5 & 11.8 \\
ESPAÑA & 0.5 & 5.1 \\
\hline
\end{tabular}

Fuente: OCDE (2012).

En cuanto al desglose de los datos españoles en las diferentes regiones del país se observa que, al igual que sucede con el resto de indicadores, existe una importante concentración geográfica, especialmente en Madrid y Cataluña (con 23 y $21 \%$ del total de solicitudes por vía nacional, respectivamente). Si a estas dos comunidades, se les añade Valencia, Andalucía y País Vasco el resultado es que, entre las cinco, aglutinan más de $75 \%$ de las patentes solicitadas en Espańa (оерм, 2010).

Finalmente, en el estudio de los resultados del SEI, resulta ilustrativo analizar algunos datos referentes a las exportaciones e importaciones de productos de alta tecnología. Esto permite hacernos una idea de la posición que ocupan en los mercados internacionales los bienes tecnológicos producidos en Espańa.

En este caso, la economía española muestra una importante dependencia exterior (gráfica vi), lo se refleja significativamente en la tasa de 
cobertura (el cociente entre exportaciones e importaciones) del comercio de productos tecnológicos. Ese ratio para 2010 muestra que las exportaciones españolas de tecnología sólo fueron $40.3 \%$ de las importaciones. Este dato se sitúa, además, muy por debajo del registrado por el conjunto del comercio exterior español, donde las exportaciones fueron $77.8 \%$ de las importaciones para ese mismo año. En otras palabras, los productos tecnológicos son menos competitivos en los mercados internacionales que el resto de las exportaciones españolas.

\section{Figura VI \\ Tasa de cobertura del comercio exterior total y de productos de alta tecnología (2001-2010)}

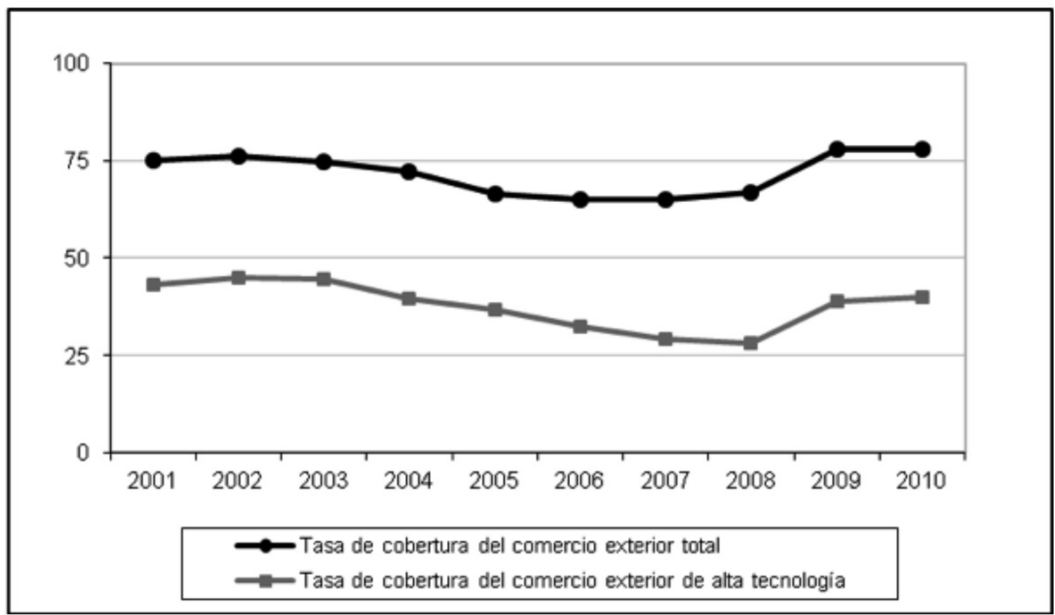

Fuente: INE (2012b).

Asimismo, el negativo saldo comercial (diferencia entre exportaciones e importaciones) de productos tecnológicos se ha ido incrementando sucesivamente, hasta llegar a situar a Espańa como el segundo país de la OCDE con mayor déficit de balanza de pagos tecnológicos y el primero en relación a su tamaño en 2003 (Molero, 2007). En los años siguientes, esta tendencia ha continuado, aunque en menor medida a partir de la crisis económica de 2008. Todo esto pone de relieve la escasa presencia de los productos tecnológicos españoles en los mercados internacionales.

\section{Conclusiones}

Del análisis realizado en este trabajo, se pueden extraer las siguientes conclusiones que presentamos a modo de diagnóstico general, que lo 
orientaremos hacia los tres aspectos del SEI contemplados en el artículo: agentes, recursos y resultados.

En primer lugar, respecto a los agentes del SEI, destaca el papel del sector público español, que se muestra como el actor con mejor desempeño innovador en términos comparativos. En el otro extremo, la implicación del sector empresarial resulta insatisfactoria: con bajos niveles de capital humano, escasa cooperación empresarial en I+D y exigua creación de negocios tecnológicos. Por ello, concluimos que las empresas son el eslabón más débil del seI.

El resto de los agentes, a pesar de sus avances en los últimos años, siguen presentando desempeños mejorables en algunos aspectos: dificultades de coordinación e ineficiencias en el marco regulatorio de las administraciones públicas; reducida dimensión y escasez de servicios en las organizaciones de soporte a la innovación, y un entorno poco favorable dada la reducida dimensión de los mercados de bienes tecnológicos, la escasez relativa de herramientas financieras para la $\mathrm{I}+\mathrm{D}$ y el bajo nivel educativo de la población.

En lo que respecta a los recursos, destaca el significativo acercamiento que se produjo durante los últimas décadas a los países de su entorno. Esto se constata tanto en términos de gastos como en recursos humanos dedicados a las actividades innovadoras. No obstante, España sigue ocupando posiciones rezagadas en estos indicadores. Además, la distribución de los recursos entre actores, refleja una reducida implicación del sector empresarial, frente a la gran presencia del sector público.

En cuanto a los resultados, las publicaciones científicas suponen el punto fuerte del SEI. En este indicador, España se sitúa relativamente cerca -aunque aún por debajo- de las economías más avanzadas, y muy por encima de la posición relativa que ocupan el resto de indicadores del SEI. En el otro lado de la balanza, los registros de patentes y las exportaciones de productos tecnológicos presentan resultados muy negativos, que son reflejo de una menguada capacidad de generación e incorporación de innovaciones a la actividad productiva y empresarial española.

Cuando se comparan las posiciones que ocupa el país en los rankings internacionales sobre recursos con las posiciones en los resultados de la actividad innovadora se puede constatar la reducida eficiencia innovadora de la economía española -el sEI presenta mejor situación en recursos que en resultados-. La diferencia entre ambas situaciones indica que el esfuerzo empleado en innovación está generando en España unos rendimientos proporcionalmente inferiores a los que se obtienen en el resto de países de su entorno.

Cabe destacar también la gran concentración geográfica de las actividades innovadoras en un número reducido de comunidades autónomas. 
Madrid y Cataluña aglutinan en torno a 50\% de los recursos y de los resultados obtenidos por el sistema. Si a estas dos regiones se les ańaden Andalucía, Valencia y País Vasco, entonces este porcentaje se sitúa en torno a $75 \%$ del total. El resto del país dedica considerablemente menos recursos a la innovación y obtiene resultados menos relevantes.

Ante este diagnóstico, las vías de mejora para las políticas de innovación son múltiples, y resulta difícil jerarquizar su urgencia; sin embargo, no queremos dejar de apuntar al menos tres recomendaciones de carácter estructural que consideramos relevantes: una profunda mejora del sistema educativo capaz de acercar al capital humano español al de los países más avanzados, una articulación eficiente del marco regulatorio en las administraciones públicas que facilite la implicación empresarial en las actividades de I+D y una apuesta clara en las organizaciones de soporte a la innovación por la colaboración entre el sector público y las empresas privadas. Los resultados de estas medidas no se reflejarán de forma inmediata, pero sin duda contribuirán a mejorar el desempeño a largo plazo del Sistema Español de Innovación.

\section{Bibliografía}

APTE (Asociación de Parques Científicos y Tecnológicos de España) (2010), "Actividad de los parques científicos y tecnológicos miembros de la APTE de 2010”, Informes de la Asociación de Parques Científicos y Tecnológicos de España, Málaga, <http://www.apte.org/ es/documents/InformeEstadisticasAPTE_def.pdf $\geq 29$ de diciembre de 2012 .

ASCRI (Asociación Española de Entidades de Capital Riesgo) (2011), "INFORME Capital Riesgo \& Private Equity en España", Asociación Española de Entidades de Capital Riesgo, Madrid.

Beraza, José María y Arturo Rodríguez (2008), “El entorno español para la creación de nuevas empresas de base tecnológica: la actividad de I+D+I en España. Una comparación internacional”, Revista de Dirección y Administración de Empresas, 15, Universidad del País Vasco, San Sebastián, pp. 9-28.

Breschi, Stefano y Malerba Franco (1997), "Sectoral systems of innovation: technological regimes, Schumpeterian dynamics and spatial boundaries", en Charles Edquist (ed.), Systems of Innovation: Technologies, Institutions and Organisations, Pinter, Londres, pp. 130-156. 
Buesa, Mikel (2003), "Ciencia y tecnología en la España democrática: la formación de un sistema nacional de innovación", Información comercial española, Ministerio de Economía y Competitividad, Madrid, pp. 235-272.

Cooke, Philip (1996), "Regional innovation systems: an evolutionary approach", en Hans-Joachim Baraczyk, Philip Cooke y Martin Heidenriech (eds.), Regional Innovation Systems, London University Press, Londres, pp. 1-18.

Cañibano, Carolina (2004), "Capital humano y sistemas de innovación: una aproximación teórica y empírica basada en el análisis del caso español", tesis doctoral, Universidad Autónoma de Madrid, Madrid.

Cañibano, Carolina y Elena Castro (2010), "El sistema español de innovación", documento de trabajo núm. 2010/09, Instituto de Gestión de la Innovación y del Conocimiento, Universidad Politécnica de Valencia, Valencia, pp. 1-58.

Edquist, Charles (1997), Systems of innovation: technologies, institutions, and organizations, Pinter, Londres.

Edquist, Charles y Björn Johnson, (1997), "Institutions and organisations in systems of innovation”, en Charles Edquist (ed.), Systems of innovation-technologies, institutions and organizations, Pinter, Londres, pp. 41-63.

Eurostat (2005), “European Innovation Scoreboard 2004”, Comisión Europea, Bruselas, <http://www.insme.org/documents/Innovation_Scoreboard_2004_EN.pdf>, 29 de diciembre de 2012.

Eurostat (2014), "Innovation Union Scoreboard”, Comisión Europea, Bruselas, <http://ec.europa.eu/enterprise/policies/innovation/ files/ius/ius-2014_en.pdf>, 2 de abril de 2014.

Freeman, Christopher (1987), Technology policy and economic performance: lessons from Japan, Pinter, Londres.

Fundación Cotec (2004), "El sistema español de innovación. Situación en 2004", Fundación Cotec para la Innovación Tecnológica, Madrid, $<$ http://www.cotec.es/index.php/publicaciones/show/id/111/ti- 
tulo/libro-blanco-2004--el-sistema-espanol-de-innovacion--situacion-en-2004/id_pagina/70/categoria_show_coleccion/Sistema +Espa $\% \mathrm{C} 3 \% \mathrm{~B} 1 \mathrm{ol}+\mathrm{de}+$ innovaci\%C3\%B3n/categoria_show_ $\mathrm{id} / 27>, 10$ de diciembre de 2012.

Fundación Cotec (2009), "Informe Cotec2009", Fundación Cotec para la Innovación Tecnológica, Madrid, <http://www.cotec.es/index. php/publicaciones/show/id/1830/titulo/informe-cotec--tecnologia-e-innovacion-en-espana--2009/id_pagina/2/categoria_show_ tema/Informes+Anuales/categoria_show_id/143>, 10 de diciembre de 2012 .

Fundación Cotec (2011), "Informe Cotec2011", Fundación Cotec para la Innovación Tecnológica, Madrid, <http://www.cotec.es/index. $\mathrm{php} /$ pagina/publicaciones/novedades/show/id/945/titulo/informe-cotec-2011--tecnologia-e-innovacion-en-espana>, 10 de diciembre de 2012.

Fundación SEPI (Sociedad Estatal de Participaciones Industriales) (2009), "Las empresas industriales en 2008. Encuesta sobre estrategias empresariales", Publicaciones de la Fundación sEPI, Madrid.

INE (Instituto Nacional de Estadística) (2012a), "Encuesta sobre innovación en las empresas", INE, Madrid, <http://www.ine.es/>, 31 de marzo de 2014.

INE (Instituto Nacional de Estadística) (2012b), "Indicadores de ciencia y tecnología”, INE, Madrid, <http://www.ine.es/>, 8 de enero de 2013.

Johnson, Björn, Charles Edquist y Bengt-Åke Lundvall (2003), “Economic development and the national system of innovation approach", ponencia presentada en First Globelics Conference, 3-6 de noviembre de 2003, Río de Janeiro.

Katz, Sylvan (2006), "Indicators for Complex Innovation Systems", Research Policy, 35, Elsevier, Toronto, pp. 893-909.

López-Facal, Javier, Unai Ugalde, Agustín Zapata y Jesús Sebastián (2006), "Dinámica de la política científica española y evolución de los actores institucionales”, en Jesús Sebastián y Emilio Muñoz (eds.), 
Radiografía de la Investigación Pública en España, Biblioteca Nueva, Madrid, pp. 21-70.

Lundvall, Bengt-Åke (1985), Product innovation and user-producer interaction, Aalborg University Press, Aalborg.

Lundvall, Bengt-Åke (1992), National systems of innovation: towards a theory of innovation and interactive learning, Pinter, Londres.

Lundvall, Bengt-Åke (2007), "National innovation system-analytical concept and development tool", Industry and Innovation, 14 (1), Taylor \& Francis, Copenhague, pp. 95-119.

Molero, José (2007), "Informe crítico sobre la innovación tecnológica en la economía española: abriendo la 'caja negra", Revista Madrid, Comunidad de Madrid, Madrid, <http://www.madrimasd.org/ revista/revista39/aula/aula1.asp>, 10 de diciembre de 2012.

Molero, José (2010), "Factores críticos de la innovación tecnológica en la economía española", Instituto Complutense de Estudios Internacionales, Universidad Complutense de Madrid, Madrid, pp. 1-23.

Molero, José (2013), “Innovación y cambio tecnológico", en José Luis García Delgado y Rafael Myro (dirs.), Lecciones de economía española, Civitas Ediciones, Madrid, pp. 99-110.

Moya, Félix y Begoña Gros (2010), Indicadores bibliométricos de la actividad cientifica de Cataluña, Universitat Oberta de Catalunya, Barcelona.

Myro, Rafael (2011), "Crisis económica y modelo productivo", Información Comercial Española, 863, Ministerio de Economía y Competitividad, Madrid, pp. 79-94.

National Science Foundation (2012), "Science and Engineering Indicators 2012", National Science Foundation, Arlington County, <http:// www.nsf.gov/statistics/seind12/>, 8 de enero de 2013.

Navarro, Mikel (2002), "La cooperación para la innovación en la empresa española desde una perspectiva internacional comparada", Economía Industrial, 346, Ministerio de Industria, Energía y Turismo, Madrid, pp. 47-66. 
Nelson, Richard (ed.) (1993), National innovation systems: a comparative analysis, Oxford University Press, Oxford.

ocDE (Organización para la Cooperación y el Desarrollo Económico) (1997), "National Innovation Systems", ocDE, París.

ocDe (Organización para la Cooperación y el Desarrollo Económico) (2008), "Main Science \& Technology Indicators", vol. 2008/2, oCDE, París.

ocde (Organización para la Cooperación y el Desarrollo Económico) (2012), "Programme for International Student Assesment", OCDE, París.

OCDE (Organización para la Cooperación y el Desarrollo Económico) (2013), "Main Science \& Technology Indicators", vol. 2011/2, OCDE, París.

oepm (Oficina Española de Patentes y Marcas) (2010), "Estadísticas de propiedad industrial (1999-2010)", овPM, Madrid, <http://www. oepm.es/>, 8 de enero de 2013.

Planas-Coll, Jordi (2005), "El papel de la empresa en la formación de los trabajadores en España", Revista de Educación, 338, Ministerio de Educación, Cultura y Deporte, Madrid, pp. 125-143.

Rivas, Carlos (2007), "Los incentivos fiscales a la innovación. Una síntesis comparada", Boletín económico del ICE, núm. 2915, Ministerio de Economía y Competitividad, Madrid, pp. 13-24.

Sternitzke, Christian (2009), "Defining triadic patent families as a measure of technological strength", Scientometrics, 81 (1), Springer, Dordrecht, pp. 91-109.

Tortosa, Enrique (2006), "La I+D en el marco autonómico", en Jesús Sebastián y Emilio Muñoz (eds.), Radiografía de la Investigación Pública en España, Biblioteca Nueva, Madrid, pp. 71-95.

Recibido: 22 de febrero de 2013. Reenviado: 28 de febrero de 2014. Aceptado: 24 de abril de 2014. 
Pablo Galaso. Español. Doctor en integración y desarrollo económico por la Universidad Autónoma de Madrid, con mención de Doctorado Europeo. Actualmente es investigador en el Instituto de Economía de la Universidad de la República (Uruguay). Además, es miembro de la Red Iberoamericana de Estudios del Desarrollo y del Innovation Business and Commerce Group, junto con otros docentes de universidades españolas e iberoamericanas; asimismo, forma parte del consejo editorial de la revista Nóesis, de la Universidad Autónoma de Ciudad Juárez. Sus líneas de investigación se centran en los estudios sobre el desarrollo económico, el capital social y las redes de innovación. Recientemente ha publicado los siguientes trabajos: "Capital social y desarrollo industrial: el caso de Prato, Italia”, Estudios regionales en economía población y desarrollo, 14, Universidad Autónoma de Ciudad Juárez, Ciudad Juárez, pp. 3-33 (2013); "Capital social y desarrollo en las redes de innovación españolas", Perspectivas. Revista de Análisis de Economía, Comercio y Negocios Internacionales, 5 (2), Universidad Autónoma de San Luis Potosí, San Luis Potosí, pp. 105-132 (2011); "El comercio mundial”, en Durán, G., J. M. García y A. Sánchez, Estructura económica y relaciones internacionales, Ibergarceta Publicaciones, Madrid, pp. 174-198 (2013); "El papel del capital social en el desarrollo. Un estudio de las redes de innovación en España”, en Gutiérrez, L. y M. Limas (coords.), Nuevos enfoques del desarrollo. Una mirada desde las regiones, Red Iberoamericana de Estudios del Desarrollo, Universidad Autónoma de Ciudad Juárez, Ciudad Juárez, pp.13-46 (2011). 